\title{
Five-Year Antimicrobial Susceptibility Trends among Bacterial Isolates from a Tertiary Health-Care Facility in Kigali, Rwanda
}

\author{
Makeda Carroll, ${ }^{1}$ Ashok Rangaiahagari, ${ }^{2,3}$ Emmanuel Musabeyezu, ${ }^{3}$ Donald Singer, ${ }^{4}$ and Onyema Ogbuagu ${ }^{5 *}$ \\ ${ }^{1}$ Department of Public Health, Philadelphia, Pennsylvania; ${ }^{2}$ Department of Microbiology, SMBT Institute of Medical Science and Research Center, \\ Nashik, India; ${ }^{3}$ King Faisal Hospital, Kigali, Rwanda; ${ }^{4}$ Fellowship of Postgraduate Medicine, London, United Kingdom; \\ ${ }^{5}$ Yale AIDS Program, Section of Infectious Diseases, Yale University School of Medicine, New Haven, Connecticut
}

\begin{abstract}
Antimicrobial resistance (AMR) is a global public health threat. There is limited information from Rwanda on AMR trends. This longitudinal study aimed to describe temporal trends of antibiotic susceptibility among common bacteria. We collated the antimicrobial susceptibility results of bacteria cultured from clinical specimens collected from inpatients and outpatients and submitted to the microbiology laboratory at King Faisal Hospital, Kigali, Rwanda, from January 1, 2009, to December 31, 2013. Differences in antimicrobial susceptibility between the first and fifth year of the study for each bacterial species was assessed using $\chi^{2}$ test. Of 5,296 isolates collected, $46.7 \%$ were Escherichia coli, $18.4 \%$ were Klebsiella spp., $5.9 \%$ were Acinetobacter spp., $7.1 \%$ were Pseudomonas spp., $11.7 \%$ were Staphylococcus aureus, and $10.3 \%$ were Enterococcus spp. Colistin and imipenem had greatest activity against gramnegative bacteria. Acinetobacter spp. showed the greatest resistance profile to antimicrobials tested, relative to other gram-negative bacteria. Vancomycin retained excellent activity against S. aureus and Enterococcus species (average susceptibility was $100 \%$ and $99.4 \%$, respectively). Trend analysis determined that resistance to imipenem increased significantly among Klebsiella, E. coli, Pseudomonas, and Acinetobacter isolates; there was also rising resistance to colistin among E. coli and Pseudomonas species. Only E. coli demonstrated increased resistance to gentamicin. For gram-positive pathogens, vancomycin susceptibility increased over time for Enterococcus species, but was unchanged for $S$. aureus. Our data suggest that resistance to imipenem and colistin are rising among gram-negative bacteria in Rwanda. Proper infection control practices and antimicrobial stewardship will be important to address this emerging threat.
\end{abstract}

\section{INTRODUCTION}

Antimicrobial resistance (AMR) is an emerging threat on a global scale, ${ }^{1,2}$ so much so that the issue has appropriately attracted the attention of international health agencies like the World Health Organization (WHO), which have developed ambitious initiatives to combat AMR. ${ }^{3-5}$ Unfortunately, and as emphasized by WHO's global report on surveillance of AMR in 2014, some of the regions of the world with high AMR rates, including sub-Saharan Africa, have generated the least data on prevalence and trends of AMR, which is typically the first step in addressing the problem..$^{6-8}$

The majority of data published on AMR in east Africa arises from Kenya and Ethiopia, whereas very little emanates from Rwanda or Burundi. ${ }^{7}$ Available data are limited in scope as they are heavily skewed toward rates observed in hospital settings, with a paucity of information on resistance in the community. ${ }^{7}$ National-level data on AMR from the region are almost nonexistent. Even where AMR rates are known, the progress of research is slow relative to the overall global effort. ${ }^{6-9}$

Data from the late 1980s and 1990s from Rwanda indicate the presence of multidrug resistance among various pathogens including Streptococcus pneumoniae, Neisseria gonorrhea, Salmonella typhimurium, and Shigella species. ${ }^{10-14}$ Subsequent reports by Muvunyi and others in 2011, showed higher rates of AMR than previously reported among uropathogenic Escherichia coli isolates, suggesting increasing trends for AMR. ${ }^{15}$ Quite alarming in that study was the observed high rate of resistance of $E$. coli to commonly used oral antibiotics

* Address correspondence to Onyema Ogbuagu, Yale AIDS Program, Section of Infectious Diseases, Yale School of Medicine, 135 College Street, Suite 323, New Haven, CT 06519. E-mail: onyema.ogbuagu@ yale.edu to treat urinary tract infections, including amoxicillin (89.3\%), ciprofloxacin $(41.3 \%)$, nitrofurantoin $(44.9 \%)$, and cotrimoxazole $(82.7 \%){ }^{15}$

More recently, a study conducted by Ntirenganya and others in 2015 , showed that $31.4 \%$ and $58.7 \%$ of E. coli and Klebsiella isolates, respectively, were resistant to at least one of the third-generation cephalosporins and 8\% of E. coli isolates were resistant to imipenem, suggesting extended-spectrum beta-lactamase (ESBL) and carbapenemase-producing strains were present. Furthermore, $82 \%$ and $6 \%$ of Staphylococcus aureus strains were noted to be oxacillin and vancomycin resistant, respectively. ${ }^{16}$

Together, these published studies from Rwanda indicate the significant presence of multidrug-resistant (MDR) pathogens, but temporal AMR trends have not been well documented. This study aims to describe 5-year trends of AMR across a broad spectrum of gram-negative and gram-positive pathogens obtained from a large tertiary health-care facility in Rwanda.

\section{MATERIALS AND METHODS}

Study design/setting. This was a longitudinal study conducted from January 1, 2009 to December 31, 2013, at King Faisal Hospital (KFH) in Rwanda. This is a large 160-bed capacity multispecialty tertiary health-care facility located in Rwanda's capital city of Kigali. Data were collected on all clinical specimens obtained from both inpatients and outpatients which yielded bacterial growth in culture during the study time frame. No identifying patient information was collected.

Sample collection and processing. Data were collected on all clinical specimens sent to the microbiology laboratory at $\mathrm{KFH}$ and included urine, blood, sputum, cerebrospinal fluid, pus swabs, and pleural and ascitic fluid specimens. Samples with improper labeling and those with inadequate patient and specimen identifiers were excluded from the study. 
Similar bacterial species isolated from different samples of the same patient were grouped as a single isolate. Antimicrobial susceptibility patterns of the following organisms were captured during the study time frame: E. coli, Klebsiella spp., Acinetobacter spp., Pseudomonas spp., S. aureus, and Enterococcus spp. A total of 5,296 bacterial isolates were obtained during the study period. Data were aggregated annually, so that the proportion of bacteria susceptible to each antibiotic was provided for each year.

Blood samples were inoculated into blood culture bottles. Urine, wound, pleural and ascitic fluid, as well as sputum cultures were collected in sterile containers. Laboratory materials including sterile containers, antimicrobial disks, and culture media, were manufactured by Becton, Dickinson and Company, Franklin Lakes, NJ.

Blood cultures were incubated in the BD BACTEC 9050 system (Becton, Dickinson and Company) at $37^{\circ} \mathrm{C}$ for 5 days. Samples with bacterial growth were subcultured on appropriate media guided by gram stain results as follows: grampositive cocci were plated on mannitol salt agar and blood agar, whereas MacConkey agar and blood agar media were used for isolation of gram-negative bacilli. Additional identification of gram-positive cocci species was performed using catalase and coagulase tests. Identification of species of gramnegative bacilli was done by colony morphology and by using API 20E diagnostic strips (bioMerieux, Hazelwood, MO).

Urine samples, after wet mount examination, were cultured on blood agar and cysteine lactose electrolyte-deficient agar. The number of colonies was counted after 18-24 hours of incubation at $37^{\circ} \mathrm{C}$. Specimens with $>10^{5}$ colony-forming units $/ \mathrm{mL}$ urine were considered to show significant growth. Maximum duration of incubation was 48 hours. For wound swabs, pleural fluid, ascites and sputum specimens, the gram stain morphology of principal pathogens dictated the selection of appropriate medium for culture, which was then incubated at $37^{\circ} \mathrm{C}$ for 24 hours. As with other specimens, identification of bacterial species was done using a combination of colony morphology, growth characteristics on selective media, and by using API 20E diagnostic strips to identify gram-negative bacilli.

Antimicrobial susceptibility testing was performed by the Kirby-Bauer disk diffusion method. The following antibiotic disks were used: ampicillin, $10 \mu \mathrm{g}$; cephalexin, $30 \mu \mathrm{g}$; ceftazidime, $30 \mu \mathrm{g}$; cefotaxime, $30 \mu \mathrm{g}$; ceftriaxone, $30 \mu \mathrm{g}$; cefalothin, $30 \mu \mathrm{g}$; cefuroxime, $30 \mu \mathrm{g}$; nalidixic acid, $30 \mu \mathrm{g}$; ciprofloxacin, $5 \mu \mathrm{g}$; levofloxacin, $5 \mu \mathrm{g}$; amikacin, $30 \mu \mathrm{g}$; amoxicillin/clavulanic acid (amox/clav), 20/10 $\mu \mathrm{g} ;$ erythromycin, $10 \mu \mathrm{g}$; gentamicin, $10 \mu \mathrm{g}$; amikacin, $30 \mu \mathrm{g}$; imipenem, $10 \mu \mathrm{g}$; norfloxacin, $10 \mu \mathrm{g}$; penicillin, 10 units; oxacillin, $1 \mu \mathrm{g}$; piperacillin, $100 \mu \mathrm{g}$; cotrimoxazole, 1.25/23.75 $\mu \mathrm{g}$; nitrofurantoin, $300 \mu \mathrm{g}$; chloramphenicol, $30 \mu \mathrm{g}$; colistin, $10 \mu \mathrm{g}$; and vancomycin, $30 \mu \mathrm{g}$.

A suspension from growth on solid media plates was prepared by adding bacterial colonies into sterile distilled water until it approximated the same turbidity as the MacFarland turbidity standard, 0.5 . The resulting suspension was inoculated on Mueller-Hinton agar by using a sterile cotton swab. After this procedure, antimicrobial disks were added to the plate with at least $20 \mathrm{~mm}$ between each disk and subsequently incubated at $37^{\circ} \mathrm{C}$ for $18-24$ hours. Interpretation of the diameter of bacterial growth inhibition was performed according to 2009 Clinical and Laboratory Standards Institute (CLSI) guidelines and subsequently, the 2012 guidelines, when they became available. Quality control for the KirbyBauer disk diffusion test was performed using three American Type Culture Collection (ATCC) strains: E. coli ATCC 25922, S. aureus ATCC 25923, and Pseudomonas spp. ATCC 27853. Suspensions of the organisms were prepared as described above, and the inhibition diameter obtained was compared with the standard range expected for the ATCC strains.

Statistical analysis. The antimicrobial susceptibility of an organism was expressed as a percentage of the total same species isolates that were sensitive to a particular antimicrobial. This was calculated on an annual basis (calendar year), and trends were assessed from year to year over the entire study period for each bacterial organism. Average annual susceptibility and standard deviation over the entire study period were calculated to provide insight on overall antimicrobial susceptibility patterns. Differences in antimicrobial susceptibility between the first and fifth year of the study for each bacterial species was assessed using $\chi^{2}$ test. Somers' D statistic $(C \mid R)$ was used to determine the significance of positive or negative trends in changes in antimicrobial susceptibility over time. Statistical software used was base SAS (R) version 9.2 (SAS institute Inc., Cary, NC).

\section{RESULTS}

Description of sample. Overall, 5,296 bacterial isolates were obtained between 2009 and 2013, including E. coli $(46.7 \%)$, Klebsiella spp. (18.4\%), S. aureus (11.7\%), and Enterococcus spp. (10.3\%). Pseudomonas spp. and Acinetobacter spp. constituted $7.1 \%$ and $5.9 \%$ of isolates, respectively (Table 1).

Average annual antimicrobial susceptibility profile. Escherichia coli was found to be most susceptible to colistin (98.6\%), imipenem (92.2\%), and nitrofurantoin (84.8\%) over the 5-year period (Table 1). It was least susceptible to ampicillin (14.8\%), piperacillin (35.4\%), and amoxicillinclavulanate $(36.0 \%)$. Ciprofloxacin and cefuroxime retained activity against $50.8 \%$ and $75.2 \%$ of $E$. coli isolates, respectively, whereas ceftriaxone and ceftazidime were active against $67.4 \%$ and $66.9 \%$ of isolates (only 2-year data available for the latter antimicrobials). Klebsiella spp. were found to be most susceptible to colistin $(99.8 \%)$, imipenem $(89.4 \%)$, and norfloxacin $(69.8 \%)$; and least susceptible to piperacillin (18.2\%), amoxicillin-clavulanate (24.6\%), ceftriaxone $(24.8 \%)$, and cotrimoxazole $(28.4 \%)$.

Colistin had activity against $81.5 \%$ and $97 \%$ of Acinetobacter spp. and Pseudomonas spp., respectively, whereas for imipenem, susceptibility rate was $45.2 \%$ and $84.3 \%$, respectively, for the same organisms. Amikacin and ciprofloxacin were more active against Pseudomonas spp. (76.8\% and $82.8 \%$ ) than Acinetobacter spp. (59.2\% and $18.4 \%$ ), respectively. Overall, imipenem, colistin, and the aminoglycosides (gentamicin and amikacin) had the most favorable susceptibility profiles for gram-negative pathogens.

Among gram-positive bacteria, susceptibility to vancomycin was $100 \%$ for $S$. aureus and $99.4 \%$ for Enterococcus spp. Oxacillin was active against $97.8 \%$ of $S$. aureus isolates, whereas the susceptibility rate for cephalexin, cotrimoxazole, and ciprofloxacin were $86.4 \%, 72 \%$, and $85 \%$, respectively. Enterococcus spp. showed low susceptibility to gentamicin $(27.0 \%)$ and levofloxacin $(54.6 \%)$, whereas ampicillin was active against $82.6 \%$ of isolates. 
TABLE 1

Average annual antimicrobial sensitivity (\%) of bacterial isolates from 2009 to 2013

\begin{tabular}{|c|c|c|c|c|c|c|}
\hline \multirow[b]{2}{*}{ Antimicrobial agent } & \multicolumn{6}{|c|}{$\begin{array}{c}\text { Bacterial species } \\
\text { Antibiotic sensitivity in \% (standard deviation) }\end{array}$} \\
\hline & $\begin{array}{l}\text { Escherichia coli } \\
\quad(N=2,473)\end{array}$ & $\begin{array}{l}\text { Klebsiella spp. } \\
\quad(N=975)\end{array}$ & $\begin{array}{l}\text { Acinetobacter spp. } \\
\quad(N=313)\end{array}$ & $\begin{array}{l}\text { Pseudomonas spp. } \\
\quad(N=375)\end{array}$ & $\begin{array}{l}\text { Staphylococcus aureus } \\
\qquad(N=617)\end{array}$ & $\begin{array}{l}\text { Enterococcus spp. } \\
\quad(N=543)\end{array}$ \\
\hline Ampicillin & $14.8(3.7)$ & - & - & - & $20.0(6.3)$ & $82.6(1.8)$ \\
\hline Penicillin & - & - & - & - & - & $26.8(12.8) \dagger$ \\
\hline Oxacillin & - & - & - & - & $97.8(1.1)$ & - \\
\hline Piperacillin & $35.4(10.1)$ & $18.2(4.0)$ & - & $77.0(6.5)$ & - & $70.4(32.2)$ \\
\hline Amoxicillin-clavulanate & $36.0(21.7)$ & $24.6(14.3)$ & - & - & $40.6(23.0)$ & $89.0(6.2)$ \\
\hline Cephalexin & - & - & - & - & $86.4(5.7)$ & - \\
\hline Cefuroxime & $75.2(6.0)$ & $48.4(17.2)$ & - & - & - & - \\
\hline Cefotaxime & - & $29.0(8.7)$ & $8.0(3.3)$ & - & - & - \\
\hline Ceftriaxone & - & $24.8(18.9)$ & $7.4(4.0)$ & - & - & - \\
\hline Ceftazidime & - & $31.6(12.5)$ & $15.4(4.0)$ & $81.0(11.4)$ & - & - \\
\hline Nalidixic acid & $51.6(5.1)$ & $54.6(12.0)$ & - & - & - & - \\
\hline Norfloxacin* & $66.4(2.9)$ & $69.8(14.3)$ & - & - & - & - \\
\hline Ciprofloxacin & $50.8(9.7)$ & $49.6(5.0)$ & $18.4(1.8)$ & $82.8(4.6)$ & $85.0(9.7)$ & - \\
\hline Levofloxacin & - & $46.0(0.0) \dagger$ & $22.6(12.7)$ & - & - & $54.6(18.4)$ \\
\hline Cotrimoxazole & $28.5(3.4)$ & $28.4(6.2)$ & - & - & $72.0(15.8)$ & - \\
\hline Nitrofurantoin* & $84.8(3.7)$ & $47.8(8.6)$ & - & - & - & - \\
\hline Chloramphenicol & - & - & - & - & - & $59.8(10.6)$ \\
\hline Erythromycin & - & - & - & - & $64.0(10.7)$ & - \\
\hline Amikacin & - & $64.8(13.4)$ & $59.2(22.4)$ & $76.8(16.3)$ & - & - \\
\hline Gentamicin & $74.0(2.6)$ & $51.8(3.8)$ & - & $77.0(9.4)$ & $87.2(6.5)$ & $27.0(6.0)$ \\
\hline Imipenem & $92.2(11.0)$ & $89.4(10.8)$ & $45.2(29.5)$ & $84.3(31.5) \dagger$ & - & - \\
\hline Colistin & $98.6(2.0)$ & $99.8(1.8)$ & $81.5(20.2) \dagger$ & $97.0(6.0) \dagger$ & - & - \\
\hline Vancomycin & - & - & - & - & $100(0.0)$ & $99.4(1.3)$ \\
\hline
\end{tabular}

$\nmid$ Less than 5 years of data; only groups with at least 3 years of data were included.

Antimicrobial susceptibility trends. The results of the $\chi^{2}$ test comparing antimicrobial susceptibility rates for each pathogen in the first (2009) and last year (2013) of the study are shown in Figure 1. The most frequent antimicrobial susceptibility trend pattern over the study period was "no trend" occurring in 29/61 (47.5\%) of organism/antimicrobial groups for which susceptibility testing was performed. Of the remaining 32 groups, the majority (22), that is, $68.8 \%$ were characterized as "negative trend," implying significant decreases in antibiotic susceptibility or increasing AMR over time. Only 10 groups were classified as having a "positive trend": Acinetobacter spp. and colistin; Klebsiella spp. and nitrofurantoin, cefotaxime, ceftriaxone, and ceftazidime; $S$. aureus and erythromycin, gentamicin, and cephalexin; Enterococcus spp. and amoxicillinclavulanate and vancomycin.

Across all bacterial isolates, E. coli had the greatest number of "negative trend" groups $(N=9)$ showing decreasing susceptibility, or increasing resistance, to amoxicillin-clavulanate, gentamicin, nalidixic acid, norfloxacin, ciprofloxacin, cefuroxime, piperacillin, imipenem, and colistin (Figure 2). Klebsiella spp. had four "negative trend" groups, including amoxicillin-clavulanate, norfloxacin, cefuroxime, and imipenem (Figure 3), whereas Acinetobacter spp. also had two negative trend groups, with decreasing susceptibility to levofloxacin, and imipenem during the study period (Figure 4). Among the group of 23 antimicrobials tested against the different bacterial species, amoxicillin-clavulanate and imipenem registered the greatest frequency of "negative trend" susceptibility profiles.

\section{DISCUSSION}

Several published studies have highlighted the growing threat of AMR in sub-Saharan Africa. ${ }^{7,8,17,18}$ It is somewhat reassuring that there is evidence that AMR is getting more attention than ever before in the region, as in recent decades there has been an increasing number of publications on the subject, ${ }^{7}$ but much more work needs to be done to effectively address the threat. Our study adds to the regional concerns on AMR as it shows that MDR gram-negative pathogens are prevalent in Rwanda and that rates of resistance to important drugs are rising. Three findings in particular are concerning and buttress the point: first is the high resistance rates of gram-negative pathogens to commonly used oral antibiotics like cotrimoxazole, ciprofloxacin, amoxicillin-clavulanate, and cefuroxime; second is the prevalence of resistance to third-generation cephalosporins by Enterobacteriaceae; and third is the significant rising trend of resistance to broad-spectrum antibiotics such as imipenem and colistin which are typically used as "salvage" antibiotics for MDR gram-negative bacterial infections.

Our findings are comparable to other similar studies from the region. Mackay and others, who conducted a 12-month AMR survey from October 2011 to September 2012 at a tertiary facility in Cape Town, South Africa, found that for health-care associated Enterobacteriaceae bloodstream isolates, susceptibility rates were $58.5 \%$ to ceftriaxone, $64.6 \%$ to gentamicin, and $70 \%$ to ciprofloxacin. The study also found that for health-care acquired Pseudomonas and Acinetobacter strains, they showed less than $80 \%$ susceptibility to all antibiotics tested except colistin. A study on AMR performed in Gabon in 2010 covering a wide variety of clinical specimens including urine cytology, blood cultures, and urethral and vaginal swabs, showed that $18 \%$ and $3-30 \%$ of cultured Klebsiella and E. coli isolates, respectively, were found to be resistant to third-generation cephalosporins. In addition, $67 \%$ of $E$. coli isolates were resistant 


\begin{tabular}{|c|c|c|c|c|c|c|c|c|c|c|c|c|c|c|c|c|c|c|c|c|c|c|c|}
\hline & 高 & 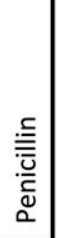 & 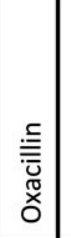 & 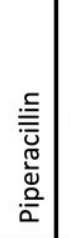 & 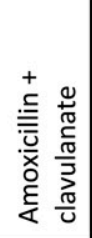 & 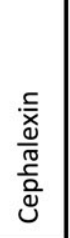 & 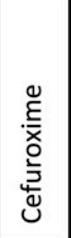 & 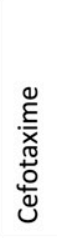 & 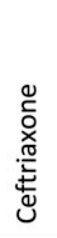 & 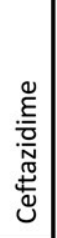 & 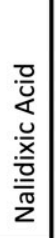 & 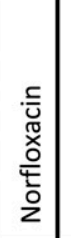 & 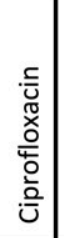 & 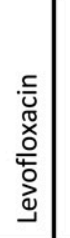 & 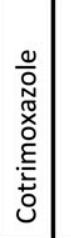 & 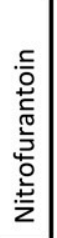 & 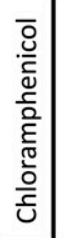 & 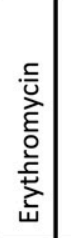 & 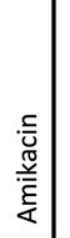 & 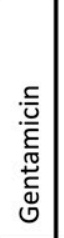 & 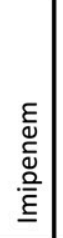 & $\frac{.}{\bar{y}}$ & 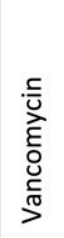 \\
\hline Escherichia coli & & & & & & & & & & & & & & & & & & & & & & & \\
\hline Klebsiella spp. & & & & & & & & & & & & & & & & & & & & & & & \\
\hline $\begin{array}{l}\text { Acinetobacter } \\
\text { spp. }\end{array}$ & & & & & & & & & & & & & & & & & & & & & & & \\
\hline $\begin{array}{l}\text { Pseudomonas } \\
\text { spp. }\end{array}$ & & & & & & & & & & & & & & & & & & & & & & & \\
\hline $\begin{array}{l}\text { Staphylococcus } \\
\text { aureus }\end{array}$ & & & & & & & & & & & & & & & & & & & & & & & \\
\hline $\begin{array}{l}\text { Enterococcus } \\
\text { spp. }\end{array}$ & & & & & & & & & & & & & & & & & & & & & & & \\
\hline
\end{tabular}

\begin{tabular}{|l|l|}
\hline \multicolumn{2}{|c|}{ Key } \\
\hline $\begin{array}{l}\text { No significant } \\
\text { difference }\end{array}$ & \\
\hline $\begin{array}{l}\text { Significant } \\
\text { difference, } \\
\text { positive trend* }\end{array}$ & \\
\hline $\begin{array}{l}\text { Significant } \\
\text { difference, } \\
\text { negative trend* }\end{array}$ & \\
\hline Not tested & \\
\hline
\end{tabular}

*P value $<0.05$

FIgURE 1. Trend in bacterial susceptibility to specific antibiotics across the study time period (2009 vs 2013) using $\chi^{2}$ test.

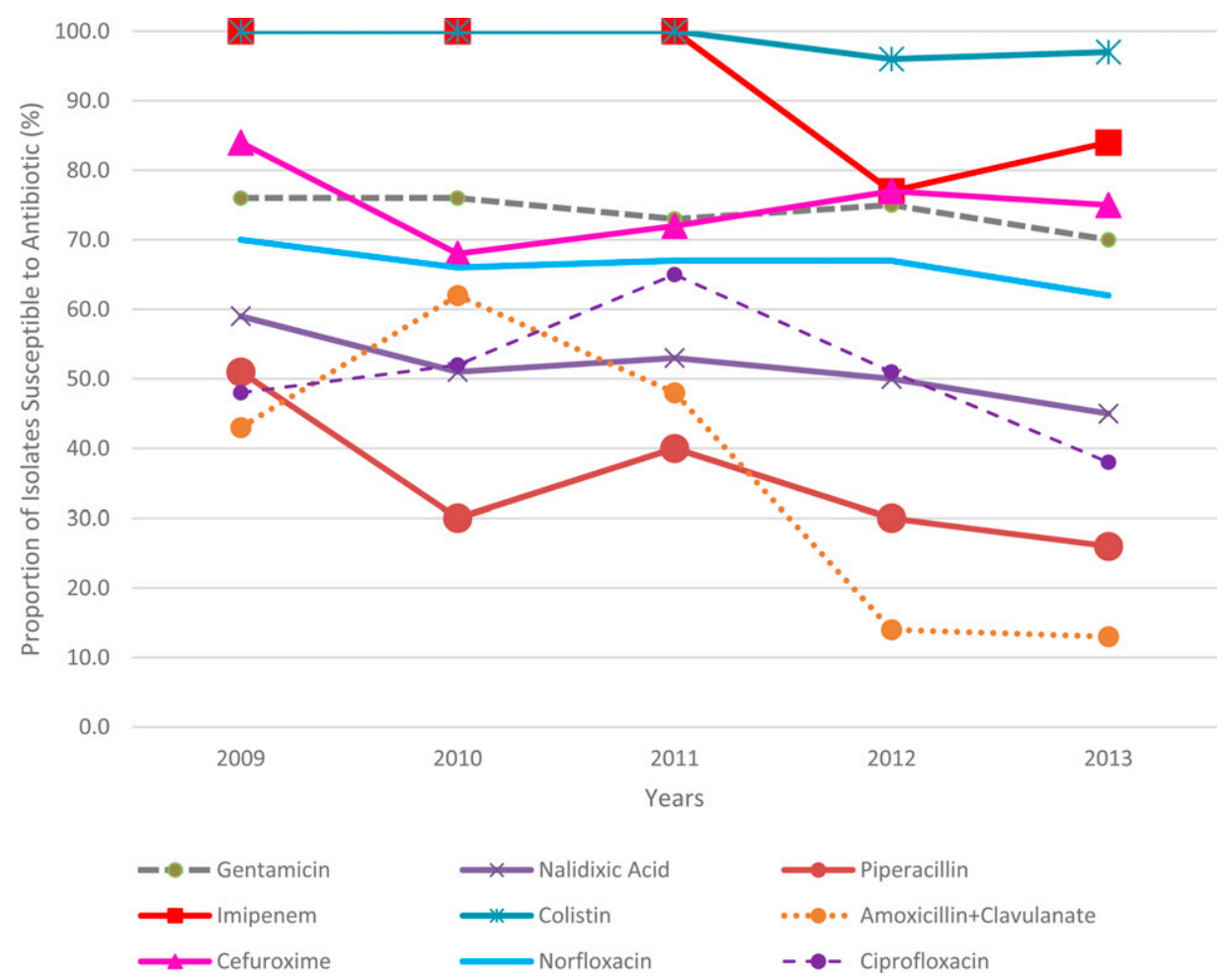

FIGURE 2. Escherichia coli susceptibility to antibiotics showing those with negative trends over time (2009-2013). 


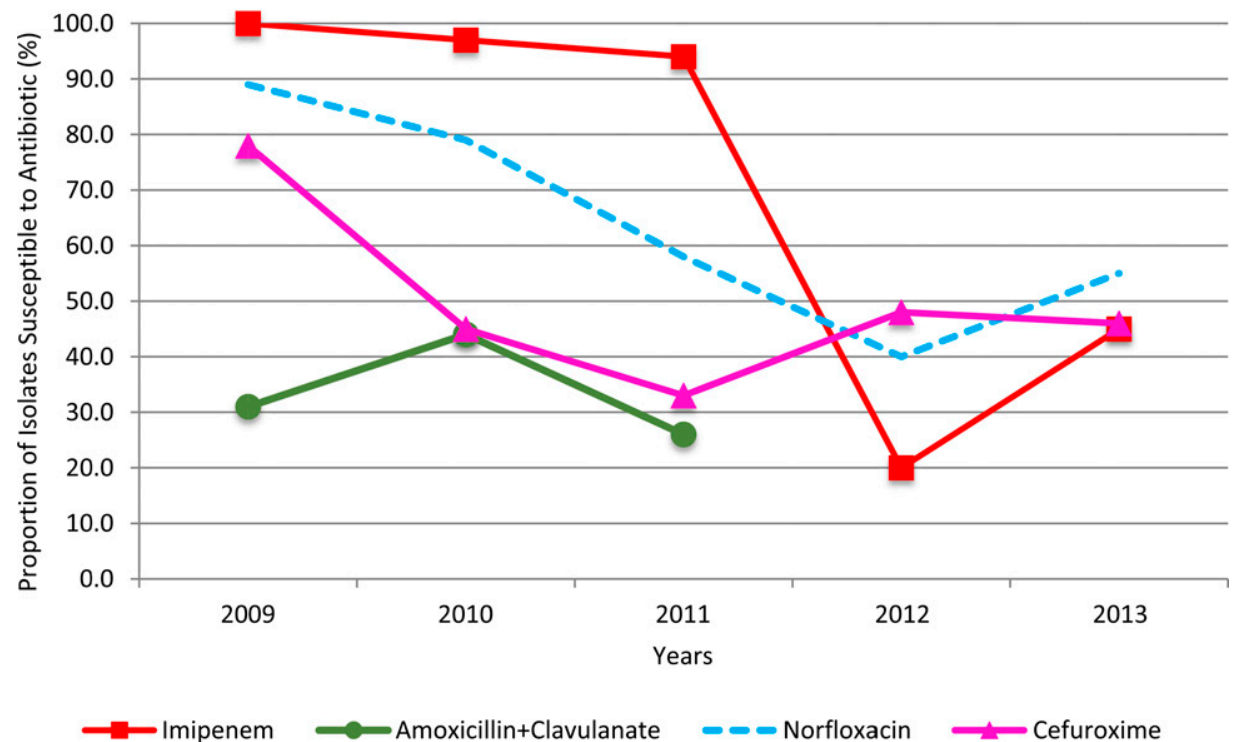

FIGURE 3. Klebsiella spp. susceptibility to antibiotics showing those with negative trends over time (2009-2013).

to amoxicillin-clavulanate, and the overall resistance rates to quinolones ranged between $58 \%$ and $78 \%{ }^{19}$

Other studies confirm the emergence of carbapenemresistant strains in sub-Saharan Africa consistent with our study findings. ${ }^{20} \mathrm{~A}$ review of published studies from Africa shows that the prevalence of carbapenemase-producing bacteria isolated in hospitals ranges from $9 \%$ to $60 \%$ in the sub-Saharan region..$^{21}$ Mushi and others reported on the prevalence of carbapenemase genes among MDR gram-negative bacilli from a tertiary hospital in Mwanza, Tanzania. They found that $35 \%$ of 227 isolates had at least one carbapenemase gene with Klebsiella pneumoniae (11\%), Pseudomonas aeruginosa $(10 \%)$, and E. coli $(8 \%)$ being the most prevalent. $^{22}$ Poirel and others published a case series in 2011 reporting seven isolates of New Delhi metalloproteinase1 -producing $K$. pneumoniae from patients on different wards of a referral hospital in Nairobi, Kenya, and were found to harbor other resistance determinants including aminoglycoside resistance genes.

One explanation for the rising trends of MDR among gram-negative bacteria could be the emergence and spread of resistant clones. Recent literature have described certain highrisk bacterial clones which harbor resistance mechanisms including plasmids such as E. coli ST131 and K. pneumoniae ST258 strains which are able to effectively colonize human hosts, have enhanced pathogenicity and may be easily transmitted within health-care settings, all features which explain their rapid spread. ${ }^{23,24}$

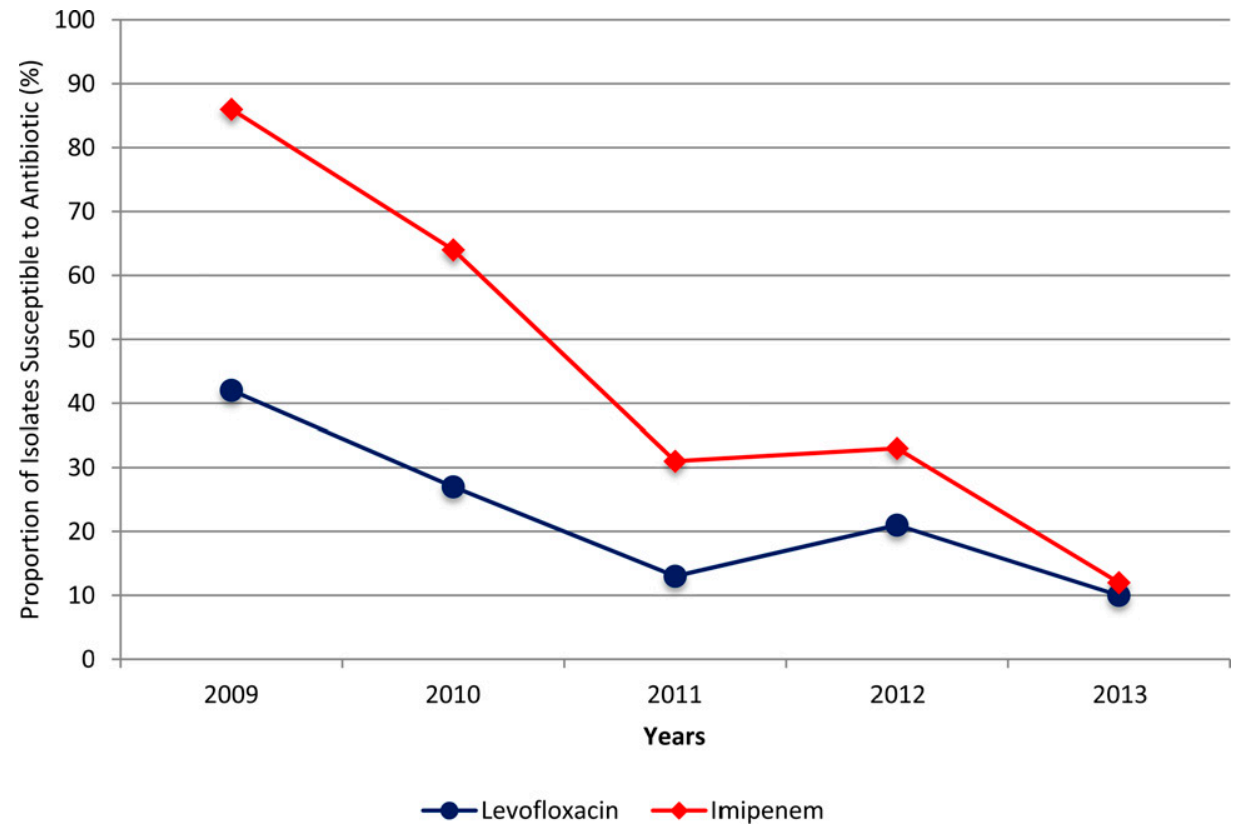

FIGURE 4. Acinetobacter spp. susceptibility to antibiotics showing those with negative trends over time (2009-2013). 
On the other hand, we did not observe any concerning negative antimicrobial susceptibility trends with $S$. aureus. Rates of susceptibility of the organism to oxacillin were high at $97.8 \%$, and there were no vancomycin-resistant isolates. This is at odds with a recent study from Rwanda which showed that $82 \%$ of $S$. aureus strains were oxacillin resistant. ${ }^{16}$ This difference may be due to differences in patient populations, the study site- $\mathrm{KFH}$ - is a privately owned tertiary health-care facility and samples were obtained from both inpatients and outpatients, whereas the site where the quoted study was performed is a public referral institution and included samples from hospitalized patients alone. One study suggests an interesting explanation for previously reported high rates of oxacillin resistance reported from lowresourced laboratories in Africa, and that is due to misidentification of coagulase-negative staphylococci as $S$. aureus, the former which is typically oxacillin resistant, due to subjective interpretation of manual phenotypic tests, specifically the tube and slide coagulation tests. ${ }^{25}$ This may be overcome by the use of automated systems.

Our study found that there were reduced susceptibility trends by Enterococcus spp. to beta-lactams-penicillin and piperacillin (average annual susceptibility $26.4 \%$ and $70.4 \%$, respectively), but $82.6 \%$ and $89 \%$ of isolates were susceptible to ampicillin and amoxicillin-clavulanate, respectively. As ampicillin has more potent activity against enterococcal species, the disparate susceptibility to the beta-lactam antibiotics was not surprising. ${ }^{26,27}$ However, some studies suggest that the observation of penicillin-resistant and ampicillinsensitive enterococcal isolates may be related to alterations in its penicillin-binding protein $4(p b p 4)$ gene. $^{28}$

A myriad of factors are to blame for high and rising AMR in sub-Saharan Africa, including Rwanda, and some are not easily tackled. Kimang'a, in an excellent article on "A situational analysis of antimicrobial resistance in Africa: are we losing the battle?"29 highlights factors contributing to AMR in Africa-including poorly qualified health-care workers who misuse antimicrobials, poor-resourced laboratories with inability to perform full antimicrobial susceptibility testing and/or produce timely results, insufficient dissemination of AMR to antibiotic prescribers, limited antimicrobial formularies, outdated or inappropriate treatment guidelines for infections, and poor infection control practices. ${ }^{29,30}$ High community access to unprescribed antimicrobials probably plays a major role. Probably underrecognized and underappreciated is the contribution of counterfeit or substandard drugs to AMR rates. ${ }^{29}$ Antimicrobial stewardship programs, supported by an ample evidence as impacting AMR, are lacking in most African health-care setings. ${ }^{31}$ It is very likely that worsening AMR trends in Rwanda reflect and are driven by the overuse of a limited number of antimicrobials which allow for the selection of resistant bacteria, and that nosocomial transmission of these organisms among patients in the setting of suboptimal infection control practices may be playing a role as well.

This study is important as it is the first of its kind in Rwanda showing AMR rates and trends over time for a broad range of bacterial pathogens and provides valuable information for health-care providers and policy makers. However, it has several limitations. The study did not include susceptibility testing for all antibiotics active against the bacteria isolated. This was due to limited resources. However, the antibiotics tested represent treatment options that are available in Rwanda. We did not perform genotyping of isolates, so could not identify any clonal outbreaks and did not confirm ESBL production by gram-negative bacteria. We did not explore differences in AMR between inpatient and outpatient specimens. As it is well known that hospitalassociated pathogens are likely to be more resistant than those that are community acquired, it is plausible that our described AMR rates are lower than would have been observed if we studied only inpatient specimens and higher than that for outpatient samples. Similarly, as we did not define the characteristics of our study population, it will be challenging to compare our reported AMR rates with other facilities within or beyond the country. As a single center study, AMR rates may also not reflect national trends. Lastly, the CLSI interpretation guidelines, changed over the time period of our study and may have negatively impacted AMR trends; however, declining susceptibility trends were already noted before the updated guidelines.

\section{CONCLUSION}

This study described prevalence and trends of AMR among common gram-positive and gram-negative bacterial pathogens in the KFH in Kigali, Rwanda. The finding of high rates of resistance by gram-negative bacteria to cephalosporins and rising rates of resistance to valuable drugs like imipenem and colistin are of concern. Institution of AMR surveillance and antimicrobial stewardship programs as well as proper infection control practices are essential to curbing this threat. Antimicrobial guidelines need to be reviewed to address the reality of decreased susceptibility to commonly used drugs.

Received May 17, 2016. Accepted for publication September 20, 2016. Published online October 31, 2016.

Acknowledgments: We wish to acknowledge the staff of the microbiology department of King Faisal Hospital (KFH), Kigali, Rwanda, for their assistance with sample processing and data collection.

Authors' addresses: Makeda Carroll, Department of Public Health, Philadelphia, PA, E-mail: makeda.carroll.13@gmail.com. Ashok Rangaiahagari, Department of Microbiology, SMBT Institute of Medical Science and Research Center, Nashik, India, E-mail: ashokrnims@gmail.com. Emmanuel Musabeyezu, King Faisal Hospital, Kigali, Rwanda, E-mail: musabeyezu@gmail.com. Donald Singer, Fellowship of Postgraduate Medicine, London, United Kingdom, E-mail: drjsinger@gmail.com. Onyema Ogbuagu, Yale AIDS Program, Section of Infectious Diseases, Yale University School of Medicine, New Haven, CT, E-mail: onyema.ogbuagu@yale.edu.

\section{REFERENCES}

1. Levy SB, 2002. The 2000 Garrod lecture. Factors impacting on the problem of antibiotic resistance. J Antimicrob Chemother 49: $25-30$.

2. Gelband H, Laxminarayan R, 2015. Tackling antimicrobial resistance at global and local scales. Trends Microbiol 23: 524-526.

3. Lusti-Narasimhan M, Pessoa-Silva CL, Temmerman M, 2013. Moving forward in tackling antimicrobial resistance: WHO actions. Sex Transm Infect 89 (Suppl 4): iv57-iv59.

4. Tapsall JW, Ndowa F, Lewis DA, Unemo M, 2009. Meeting the public health challenge of multidrug- and extensively drugresistant Neisseria gonorrhoeae. Expert Rev Anti Infect Ther 7: 821-834.

5. Shallcross LJ, Davies SC, 2014. The World Health Assembly resolution on antimicrobial resistance. J Antimicrob Chemother 69: 2883-2885. 
6. Shears P, 2001. Antibiotic resistance in the tropics. Epidemiology and surveillance of antimicrobial resistance in the tropics. Trans R Soc Trop Med Hyg 95: 127-130.

7. Omulo S, Thumbi SM, Njenga MK, Call DR, 2015. A review of 40 years of enteric antimicrobial resistance research in eastern Africa: what can be done better? Antimicrob Resist Infect Control 4: 1 .

8. Okeke IN, Aboderin OA, Byarugaba DK, Ojo KK, Opintan JA, 2007. Growing problem of multidrug-resistant enteric pathogens in Africa. Emerg Infect Dis 13: 1640-1646.

9. Perovic O, Singh-Moodley A, Duse A, Bamford C, Elliott G, Swe-Han KS, Kularatne R, Lowman W, Whitelaw A, Nana T, Wadula J, Lekalakala R, Saif A, Fortuin De-Smit M, Marais E, 2014. National sentinel site surveillance for antimicrobial resistance in Klebsiella pneumoniae isolates in South Africa, 2010-2012. S Afr Med J 104: 563-568.

10. Bogaerts J, Verhaegen J, Munyabikali JP, Mukantabana B, Lemmens P, Vandeven J, Vandepitte J, 1997. Antimicrobial resistance and serotypes of Shigella isolates in Kigali, Rwanda (1983 to 1993): increasing frequency of multiple resistance. Diagn Microbiol Infect Dis 28: 165-171.

11. Bogaerts J, Lepage P, Taelman H, Rouvroy D, Batungwanayo J, Kestelyn P, Hitimana DG, Van de Perre P, Vandepitte J, Verbist L, Verhaegen J, 1993. Antimicrobial susceptibility and serotype distribution of Streptococcus pneumoniae from Rwanda, 1984-1990. J Infect 27: 157-168.

12. Van Dyck E, Karita E, Abdellati S, Dirk VH, Ngabonziza M, Lafort Y, Laga M, 2001. Antimicrobial susceptibilities of Neisseria gonorrhoeae in Kigali, Rwanda, and trends of resistance between 1986 and 2000. Sex Transm Dis 28: 539-545.

13. Bogaerts J, Van Dyck E, Mukantabana B, Munyabikali JP, Martinez Tello W, 1998. Auxotypes, serovars, and trends of antimicrobial resistance of Neisseria gonorrhoeae in Kigali, Rwanda (1985-93). Sex Transm Infect 74: 205-209.

14. Lepage $\mathrm{P}$, Bogaerts J, Van Goethem C, Hitimana DG, Nsengumuremyi F, 1990. Multiresistant Salmonella typhimurium systemic infection in Rwanda. Clinical features and treatment with cefotaxime. J Antimicrob Chemother 26 (Suppl A): 53-57.

15. Muvunyi CM, Masaisa F, Bayingana C, Mutesa L, Musemakweri A, Muhirwa G, Claeys GW, 2011. Decreased susceptibility to commonly used antimicrobial agents in bacterial pathogens isolated from urinary tract infections in Rwanda: need for new antimicrobial guidelines. Am J Trop Med Hyg 84: 923-928.

16. Ntirenganya C, Manzi O, Muvunyi CM, Ogbuagu O, 2015. High prevalence of antimicrobial resistance among common bacterial isolates in a tertiary healthcare facility in Rwanda. Am J Trop Med Hyg 92: 865-870.

17. Kimang'a A, 2012. A situational analysis of antimicrobial drug resistance in Africa: are we losing the battle? Ethiop J Health Sci 22: 135-143.

18. Center for Disease Dynamics, Economics, and Policy, 2011. Situation Analysis: Antibiotic Use and Resistance in South Africa. Global Antibiotic Resistance Partnership, 549-596.

19. Kouegnigan Rerambiah L, Ndong JC, Mbakob Mengue Massoua P, Medzegue S, Elisee-Ndam M, Mintsa-Ndong A,
Djoba Siawaya JF, 2014. Antimicrobial profiles of bacterial clinical isolates from the Gabonese National Laboratory of Public Health: data from routine activity. Int J Infect Dis 29: $48-53$.

20. Sekyere JO, Govinden U, Essack S, 2015. The molecular epidemiology and genetic environment of carbapenemases detected in Africa. Microb Drug Resist 22: 59-68.

21. Manenzhe RI, Zar HJ, Nicol MP, Kaba M, 2015. The spread of carbapenemase-producing bacteria in Africa: a systematic review. J Antimicrob Chemother 70: 23-40.

22. Mushi MF, Mshana SE, Imirzalioglu C, Bwanga F, 2014. Carbapenemase genes among multidrug resistant gram negative clinical isolates from a tertiary hospital in Mwanza, Tanzania. BioMed Res Int 2014: 303104.

23. Mathers AJ, Peirano G, Pitout JD, 2015. The role of epidemic resistance plasmids and international high-risk clones in the spread of multidrug-resistant Enterobacteriaceae. Clin Microbiol Rev 28: 565-591.

24. Price LB, Johnson JR, Aziz M, Clabots C, Johnston B, Tchesnokova V, Nordstrom L, Billig M, Chattopadhyay S, Stegger M, Andersen PS, Pearson T, Riddell K, Rogers P, Scholes D, Kahl B, Keim P, Sokurenko EV, 2013. The epidemic of extended-spectrum-beta-lactamase-producing Escherichia coli ST131 is driven by a single highly pathogenic subclone, H30-Rx. MBio 4: e00377-13.

25. Omuse G, Kabera B, Revathi G, 2014. Low prevalence of methicillin resistant Staphylococcus aureus as determined by an automated identification system in two private hospitals in Nairobi, Kenya: a cross sectional study. BMC Infect Dis 14: 669.

26. Predari SC, Gutierrez MA, Ribas C, Molinari GS, Santoianni JE, 1991. Susceptibility of Enterococcus faecalis to twelve antibiotics, time-kill assays, and high-level aminoglycoside resistance in a university hospital in Argentina. Rev Argent Microbiol 23: 67-78.

27. Weinstein MP, 2001. Comparative evaluation of penicillin, ampicillin, and imipenem MICs and susceptibility breakpoints for vancomycin-susceptible and vancomycin-resistant Enterococcus faecalis and Enterococcus faecium. J Clin Microbiol 39: $2729-2731$.

28. Conceicao N, da Silva LE, Darini AL, Pitondo-Silva A, de Oliveira AG, 2014. Penicillin-resistant, ampicillin-susceptible Enterococcus faecalis of hospital origin: pbp 4 gene polymorphism and genetic diversity. Infect Genet Evol 28: 289-295.

29. Kimang'a AN, 2012. A situational analysis of antimicrobial drug resistance in Africa: are we losing the battle? Ethiop J Health Sci 22: $135-143$.

30. Whitelaw AC, 2015. Role of infection control in combating antibiotic resistance. S Afr Med J 105: 421.

31. Ramsamy Y, Muckart DJ, Han KS, 2013. Microbiological surveillance and antimicrobial stewardship minimise the need for ultrabroad-spectrum combination therapy for treatment of nosocomial infections in a trauma intensive care unit: an audit of an evidence-based empiric antimicrobial policy. S Afr Med J 103: 371-376. 